\title{
Entrelacs
}

Cinéma et audiovisuel

Nouvelles formes audiovisuelles documentaires

\section{Webdocumentaire et ressources numériques applicables à la mise en scène, à la délinéarisation et à l'interactivité.}

Bruno Bernard

\section{(2) OpenEdition \\ Journals}

Electronic version

URL: http://journals.openedition.org/entrelacs/1754

DOI: $10.4000 /$ entrelacs. 1754

ISSN: 2261-5482

Publisher

Éditions Téraèdre

\section{Electronic reference}

Bruno Bernard, « Webdocumentaire et ressources numériques applicables à la mise en scène, à la délinéarisation et à l'interactivité. », Entrelacs [Online], 12 | 2016, Online since 14 January 2016, connection on 01 May 2019. URL : http://journals.openedition.org/entrelacs/1754 ; DOI : 10.4000/ entrelacs.1754

This text was automatically generated on 1 May 2019.

Tous droits réservés 


\title{
Webdocumentaire et ressources numériques applicables à la mise en scène, à la délinéarisation et à l'interactivité.
}

\author{
Bruno Bernard
}

1 Forme récente du documentaire hybridé avec le web, le webdocumentaire offre aux auteurs de nouvelles ressources en leur permettant d'étendre et de déplacer les écritures «classiques » jusqu'alors mises en œuvre, et aux destinataires, de disposer d'un statut propre à l'internaute. Au-delà de ce cadre initial, nous nous proposons de repérer certaines particularités des webdocumentaires, mais aussi d'appréhender leur inscription dans le prolongement des médias qui ont, depuis les années 1980, intégré la forme numérique. La production de signes visuels dédiés au traitement d'un thème, sous une forme hypermédia, se situe aussi au centre de nos préoccupations. La construction des images « résultantes », de la narration et de l'interactivité constitue autant de vecteurs significatifs pour innover et découvrir de nouvelles voies à la représentation du réel et à sa mise en forme discursive.

\section{Qu'est-ce qu'un webdocumentaire?}

2 Afin d'appréhender les tendances des écritures filmiques du webdocumentaire, nous avons envisagé d'entreprendre un visionnage de documents mis en ligne. La consultation de plusieurs sites web dédiés à ce genre filmique nous conduit à effectuer le constat suivant: il existe un grand nombre de variantes ou de développements de documents s'inscrivant dans cette catégorie. Les résultats de cette première approche n'étant pas suffisamment significatifs, nous proposons donc de compléter notre investigation. Cette seconde approche est plus générale et retient pour point de départ la prise en compte d'une spécificité de la forme numérique. Lorsque nous considérons successivement les 
moyens d'expression accueillant un automatisme (photographie, cinéma et vidéo), nous constatons un accroissement du niveau de contrôle de la formation de l'image et de l'objet image, la forme numérique induisant le degré le plus élevé du contrôle.

Nous allons également tenter de repérer des spécificités du webdocumentaire en considérant les incidences de la forme numérique sur les deux dimensions, le web et le documentaire. D'un côté, le web apporte des ressources liées à un accès aux réseaux informatiques et sociaux alors que, d'un autre côté, les composantes image et narration $\mathrm{du}$ documentaire sont principalement contrôlées par les fonctions de génération, de modification, d'hybridation, de délinéarisation et d'interactivité.

4 Nous proposons donc de poursuivre notre approche en privilégiant ces spécificités, afin de repérer des vecteurs d'innovation des écritures filmiques interactives.

\section{Articulation de deux approches : le visionnage et les incidences de la forme numérique}

5 Lors du visionnage, nous constatons qu'un nombre conséquent d'auteurs de documents semble retenir le web comme vecteur de diffusion, en privilégiant le développement d'une interactivité basée sur un contenu linéaire segmenté pour le besoin d'intégration d'un média proposant un lien vers chacun des segments. Dans cette option, les écritures sont classiques; les images et les montages sont très proches des solutions linéaires diffusées par le média télévisuel. D’autres documents dont les auteurs ont intégré des formes plus complexes de développement de l'interactivité présentent différentes solutions pour articuler la délinéarisation et l'interactivité, afin de générer des écritures où les internautes sont plus impliqués.

6 La lecture et la consultation de tous ces documents nous amènent à penser aux années 1990, à l'âge d'or des documents interactifs diffusés via le support CD-Rom. Cependant, nous remarquons des évolutions notables relatives à l'accès aux réseaux, à l'intégration et à la fluidité des objets vidéos. Ces composantes deviennent alors les vecteurs supports des écritures. L'objectif que nous nous sommes fixé nous conduit néanmoins à ne pas nous arrêter à cette observation et à rechercher d'autres vecteurs potentiellement porteurs d'innovation.

7 Si nous rapprochons le fait que le webdocumentaire est un genre récent (2007-2008) et notre inclinaison à penser à la création de CD-Rom interactif, nous sommes amenés à considérer un type d'images porteur d'une modernité, datant de la même époque : les images de synthèse $2 \mathrm{D}$ et $3 \mathrm{D}$. L'élaboration de cette passerelle nous permet de nous souvenir que l'intégration du numérique par les images photoniques et la morphogenèse algorithmique ont favorisé l'émergence d'attentes relatives à la création de "nouvelles images ${ }^{1} »$ et de réflexions tentant de circonscrire les tendances suivies par les créateurs.

8 Ainsi sommes-nous enclins à rappeler succinctement les propositions pertinentes relatives aux images numériques, énoncées par Françoise Holtz-Bonneau. Cet auteur investit la création infographique en proposant trois modes : la composition, la mutation et la déambulation ${ }^{2}$. Nous constatons que la définition de ces trois modes offre la possibilité d'appréhender les webdocumentaires, mais les perspectives de cette approche ne correspondent que partiellement à notre projet. En effet, les vecteurs proposés s'inscrivent dans un cadre spécifique privilégiant principalement les images de synthèse où apparaissent des formes géométriques élémentaires, dont le sens est très réduit par 
rapport à celui véhiculé par des images photoniques représentant une scène réelle. Ensuite, par leurs assemblages, ces formes constituent des objets porteurs de sens.

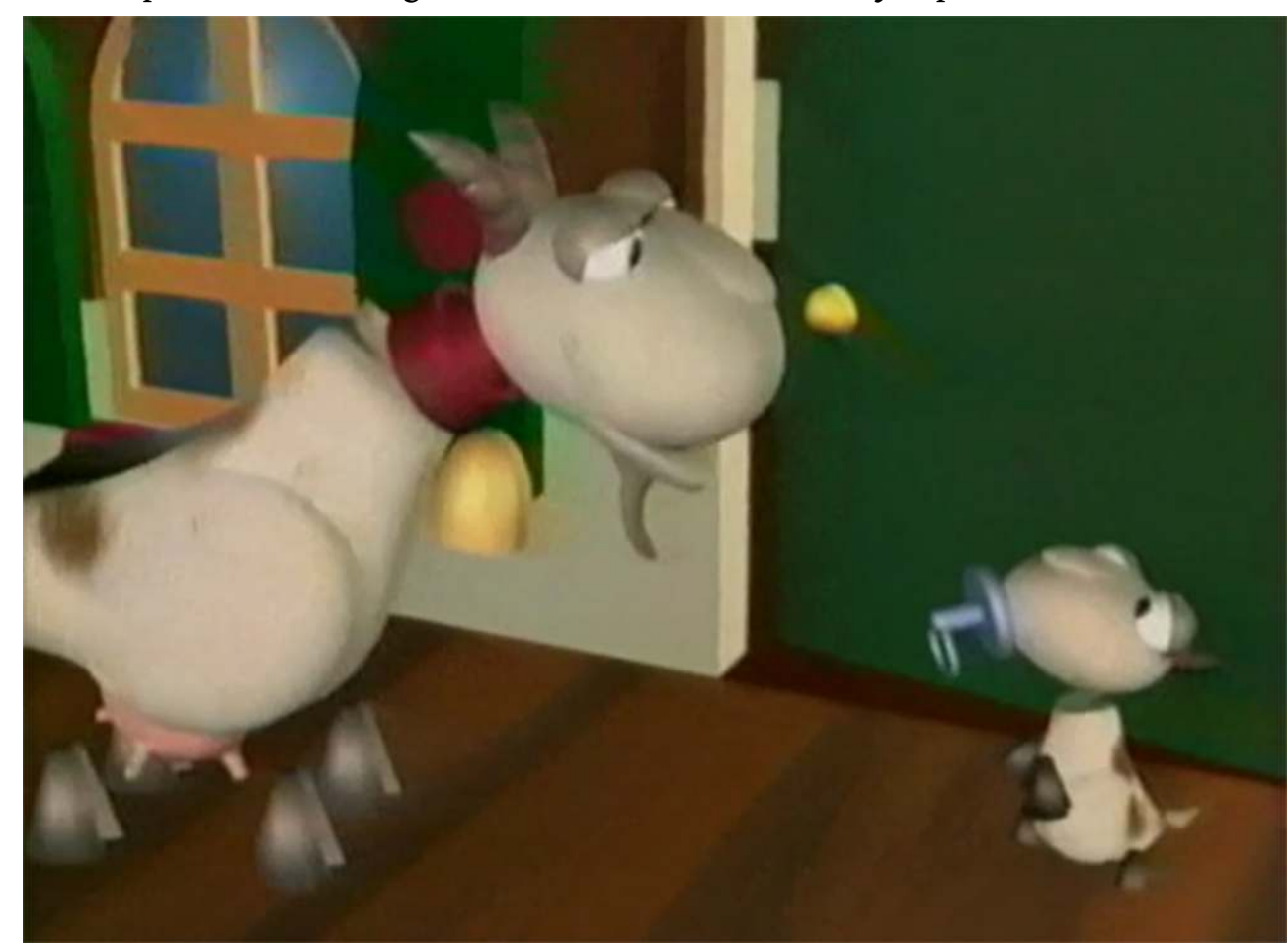

Figure 1 : Le loup, la chèvre et le chevreau, Jean de La Fontaine / « Les fables géométriques »

9 Par ailleurs, les exemples retenus par l'auteur correspondent généralement à des images " entières ${ }^{3}$ ». Dans celles-ci, les éléments convoqués sont les parties d'un objet ou d'une scène et après l'application d'un processus de composition, les éléments sont regroupés sans introduire d'écarts sémantiques entre eux ou entre les différentes parties du tout constitué. L'image résultante occupe toute la surface de l'écran télévisuel ou de la fenêtre où s'opère l'affichage, dans le cas d'un lecteur informatique. Alors que dans le webdocumentaire intégrant les ressources numériques dans une perspective innovante, au contraire, les parties constituant un ensemble visuel laissent visible l'assemblage. La frontière et les limites de chaque fragment peuvent alors être le lieu d'existence d'écarts, qui révèlent la structure de l'ensemble et participent à la construction du récit. Cette dernière remarque constitue une perspective au sein de laquelle nous positionnons notre approche.

10 Ces trois modes sont globaux et permettent d'étudier les résultats liés à l'application d'un processus de création numérique. Tout en validant leur effectivité, nous observons que la priorité accordée aux éléments d'une image "entière " correspond partiellement à l'approche des webdocuments. Dans la perspective de notre investigation, cette option ne nous semble pas suffisante et nous proposons donc de focaliser notre attention sur les éléments sources convoqués pour produire une image résultante ${ }^{4}$.

11 Afin de préciser notre proposition, nous allons revenir à la prise en compte des spécificités de la forme numérique dans un processus de création ${ }^{5}$ d'image. Ces spécificités ont pour origine le contrôle des pixels de la matrice-image, donc a fortiori le contrôle de la surface de l'image photonique et algorithmique gérée au cours des différentes phases du processus de réalisation. Si le contrôle du pixel autorise une modification de ses paramètres (luminance, chrominance), il permet aussi d'intervenir 
sur la surface produite par la juxtaposition de pixels. Avant de préciser de quelle manière nous allons découvrir que le contrôle de la surface est au centre de nos préoccupations, intéressons-nous à la représentation filmique du réel, dimension centrale du webdocumentaire. Depuis un siècle, le cinéma puis la vidéo, s'élaborent en produisant des plans filmiques, extraction spatio-temporelle du réel. Les écritures filmiques se développent donc sur la base de la saisie et de l'assemblage-montage de fragments. Si la fragmentation constitue un aspect fondamental de la représentation filmique et du découpage, elle le sera aussi dans le processus de contrôle numérique de l'image. Nous orientons donc notre investigation en direction du fragment, élément de base des écritures numériques, de la même manière que le pixel est l'élément de base de l'image numérique. Le rapprochement opéré entre le pixel et le fragment, tous deux éléments de surface, nous permettra de passer des fonctions de contrôle de l'un à la représentation et à la production de sens lié à l'autre.

\section{Le contrôle de la surface de l'image}

Après le visionnage de l'ensemble des documents filmiques de notre corpus, nous sommes en mesure d'énoncer le constat suivant: la majeure partie des auteurs développant des narrations interactives sous la forme d'un webdocumentaire privilégie une dominante. Celle-ci correspond à la gestion des deux dimensions de la surface de l'image, où s'affichent les éléments visuels convoqués simultanément pour former l'image résultante, au sein de laquelle l'internaute découvrira les différents étapes et instants de la narration ou de l'enquête, objet de son investissement. Cette observation est en relation directe avec la notion de contrôle de la surface de l'image induit par la forme numérique.

Si, d'un côté, le contrôle favorise la création de fragments en tant qu'éléments-sources, il implique par ailleurs le développement $\mathrm{du}$ «multiple» dans les images et dans les processus. Nous allons repérer les différentes déclinaisons du multiple.

La première forme évoquée est le multifenêtrage. Elle correspond à la production de zones indépendantes distribuées sur la surface de l'image. La fenêtre est donc le lieu privilégié d'accueil d'un fragment. Lors de la combinaison des différents «multiples» pour produire des éléments d'écriture, nous noterons que la fenêtre est un espace qui cache en même temps qu'il révèle. Mais aussi qu'une présence simultanée des fenêtres démultiplie les effets créés, et donc les vecteurs d'introduction et de développement de la narration et de l'interactivité.

La deuxième forme est directement liée au plan ${ }^{6}$. Le multiplan résulte de la présence de plusieurs plans dans la construction de l'image ${ }^{7}$ résultante. La juxtaposition de plans introduit dans l'ensemble crée autant de possibilités supplémentaires pour accueillir des fragments dont le positionnement sera éventuellement source de superposition. Nous évoquerons aussi le fait que le passage d'un à plusieurs plans constitue un vecteur d'introduction de la variable contrôle de la profondeur de l'espace, donc le passage d'une surface à un volume.

Initialement, le multicouche ${ }^{8}$ faisait exclusivement référence au processus de création d'une image. Il était donc réservé aux auteurs et ne concernait pas les spectateurs. Cependant, la déclinaison du multicouche dans la phase de diffusion-consultation caractérisée par la circulation entre les plans et les fenêtres est envisageable. Dans un tel cas, la prise en compte de la composante temporelle nous autorise à envisager le 
multicouche en tant que composante de la navigation à l'intérieur d'une image résultante.

\section{La présence des « multi » dans les webdocumentaires du corpus}

Dans de nombreux documents, nous repérons l'affichage de différents types d'objets visuels, dont la présence participe à la construction de l'image et de la dimension hypertextuelle du document filmique. Une image de fond, souvent photographique, accueille en incrustation des éléments textuels linguistiques, un fragment d'image iconique ou encore l'interface d'un lecteur audio. Dans les différents cas rencontrés, les fragments visuels sont mis en page et en scène, afin de présenter l'ensemble des options disponibles à des instants clés. La composition bidimensionnelle est au service de l'instant comme les éléments d'une page imprimée ou d'une page d'un site internet. Ainsi, à partir de la découverte de la surface de l'image résultante, l'internaute choisira ses options pour créer le parcours correspondant à la progression du thème développé par le webdocumentaire. A cette découverte de la surface, s'ajoute la succession des instants caractérisés par le choix d'une branche de la structure interactive, afin de passer à l'étape suivante, différenciée de la précédente par une évolution de l'affichage. Une composition surfacique remplaçant une autre composition surfacique, la surface s'articule avec la composante temporelle. Le multifenêtrage distribué sur un plan est la forme dominante rencontrée dans les documents du corpus.

Parmi l'ensemble des webdocumentaires de notre corpus, nous retiendrons en particulier Prison Valley ${ }^{9}$. Cette enquête journalistique dans l'environnement économique et social d'une région dont l'activité est centrée sur l'implantation de prisons publiques et privées, est scindée en plusieurs fragments accueillant chacun une ou deux interviews. Pendant le déroulement d'une séquence descriptive, des photographies, des plans filmiques d'espaces urbains, carcéraux et désertiques, ainsi qu'une voix off dont la traduction est incrustée dans l'image, sont proposés aux internautes. A la fin de chaque séquence, deux ou trois options de parcours sont envisageables : 1) compléter sa connaissance du sujet abordé dans la séquence (par exemple: "visiter la prison du shérif ", « débattre sur le travail des prisonniers »), 2) retourner au motel où d'autres compléments d'informations sont disponibles (par exemple : « indices-souvenirs et documents», « calepin-contacter un personnage ", "forum-discuter en direct») et 3) "continuer sur la route». Nous remarquons que l'interactivité retenue pour construire ce webdocumentaire est simple mais efficace. L'enquête propose une progression linéaire émaillée de pauses éventuelles dont l'interruption nous ramènera à l'endroit initialement quitté. Notre intérêt pour ce document réside dans la mise en scène de certaines parties, qui intègrent des potentialités induites par la forme numérique. Le multifenêtrage est retenu pour juxtaposer les fragments d'une même image, pour rapprocher le contenu de deux ou trois sources visuelles ${ }^{10}$ (Figure 2). Nous observerons que la surface de l'image résultante est toujours remplie et que parfois, la variation de la hauteur des fenêtres génère un rythme dans la composition finale. A d'autres moments, la composante temporelle est retenue pour contrôler l'introduction des fragments dans la cadre de l'image d'accueil. Des déplacements horizontaux et verticaux révèlent donc des éléments visuels qui parfois termineront leur course dans l'image ou bien la commenceront pour sortir du cadre. Les dimensions des fenêtres et l'orientation de leur base sont aussi soumises au changement. 
L'ensemble des solutions retenues est varié et inséré dans chaque partie du webdocumentaire. Complémentarité, télescopage, juxtaposition sont autant de possibilités pour s'adresser au public, pour l'interpeller, pour maintenir son attention en renouvelant aussi le contrôle du rythme de l'ensemble créé. Cependant nous remarquerons que si la gestion du fenêtrage est au service de la construction visuelle, elle s'inscrit peu dans le développement de l'interactivité.

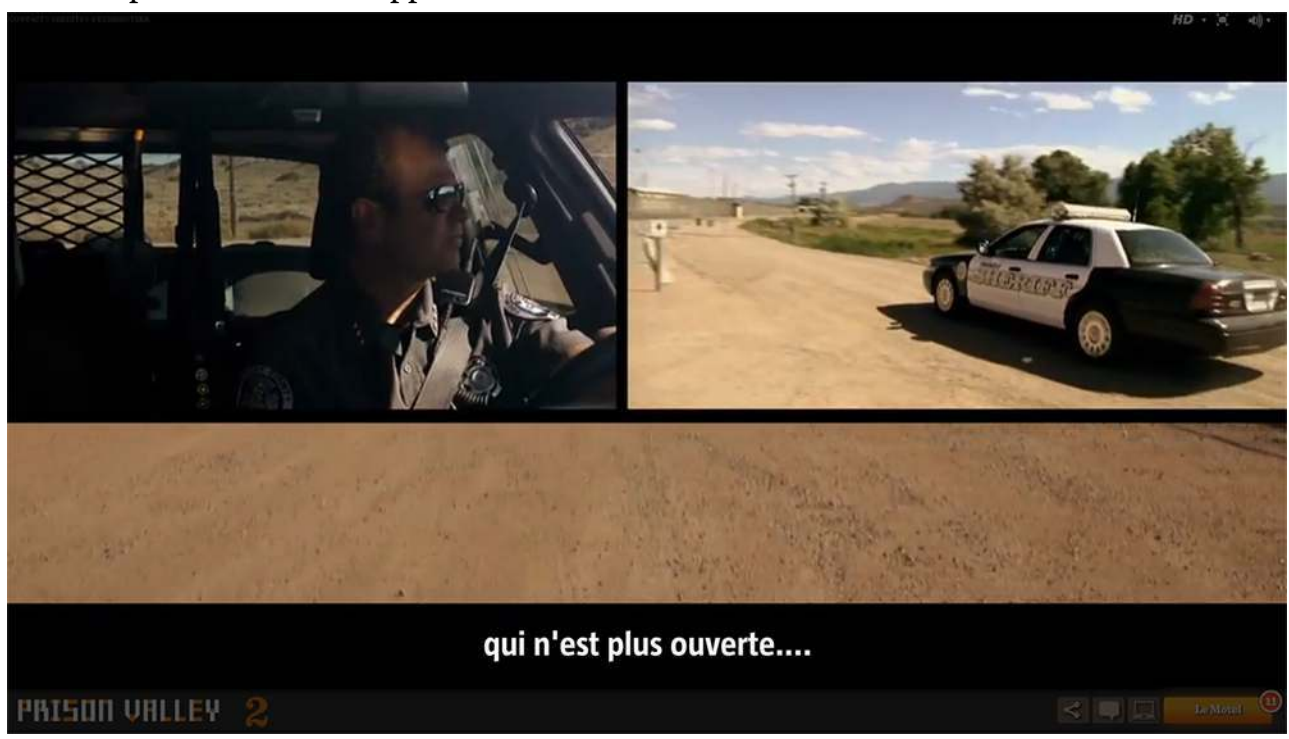

Figure 2 : multifenêtrage / "Prison Valley »

\section{Convergence des « multi » et de la troisième dimension}

L'une des problématiques de la représentation visuelle tant picturale que photonique et algorithmique, a été l'intégration de la troisième dimension absente du support d'affichage de l'image et cependant constitutive de l'espace-temps réel. De la perspective du Quattrocento jusqu'à l'immersion dans un univers virtuel simulé, la quête du réalisme a convoqué de multiples solutions. Dans le cas présent, nous nous intéressons à la troisième dimension, non pas pour atteindre un résultat tendant à se rapprocher d'un référent tridimensionnel, mais comme composante d'un document dont l'hypertextualité et l'interactivité sont des vecteurs le différenciant du documentaire.

Afin de préciser notre approche intégrant les trois «multi» et la composante "tridimension ${ }^{11}$ ", nous proposons d'en présenter les différents éléments constitutifs. Notre proposition s'inscrit dans le prolongement de l'existant et nous en abordons le premier élément en considérant les formes actuelles mises en œuvre dans les documents du corpus. Comme nous l'avons indiqué précédemment, la surface est au centre des structures organisationnelles de la distribution des signes et des fragments visuels. L'investigation de la surface nous rappelle que nous pouvons retenir l'application d'une grille $^{12}$ pour distribuer les différents éléments de la mise en image. Limitée à cette observation, la convocation de l'outil grille présente un intérêt restreint . En effet, notre propos n'a pas pour but l'étude de ce qui est actuellement réalisé pour repérer les types de grilles récurrentes. Nous retenons la grille comme vecteur structurant d'une première surface accueillant des images ou des plans filmiques dans des fenêtres. Nous la 
dénommons "surface-grille». Ensuite nous considérons la déclinaison de ce mode de structuration appliqué à plusieurs plans, c'est-à-dire à plusieurs fenêtres distribuées sur l'axe de la profondeur, soit un assemblage pouvant rappeler la création d'une troisième dimension. La structure obtenue est nommée «volume-grille» (Figure 3). Cependant, nous rappelons que l'objectif assigné à cette construction ne réside pas dans la distribution de fragments visuels sur les différents plans, afin de simuler une profondeur spatiale. Cette option est certes envisageable, mais elle limiterait le champ des possibilités que nous envisageons d'aborder.

21 Ainsi pouvons-nous disposer de plusieurs plans superposés sur lesquels des grilles différentes seront appliquées. Chaque «surface-grille » constituera un espace différencié pour accueillir des fragments visuels. Cette superposition induite par le « volume-grille» a pour conséquence le fait que le renouvellement d'un plan filmique n'entraîne pas l'affichage d'une nouvelle image dont la surface correspond à la surface globale de la fenêtre, une image en chassant une autre. Mais aussi, l'imbrication des éléments visuels des plans implique la possibilité de proposer une évolution du contenu suivant un ordre de succession et/ou de simultanéité.

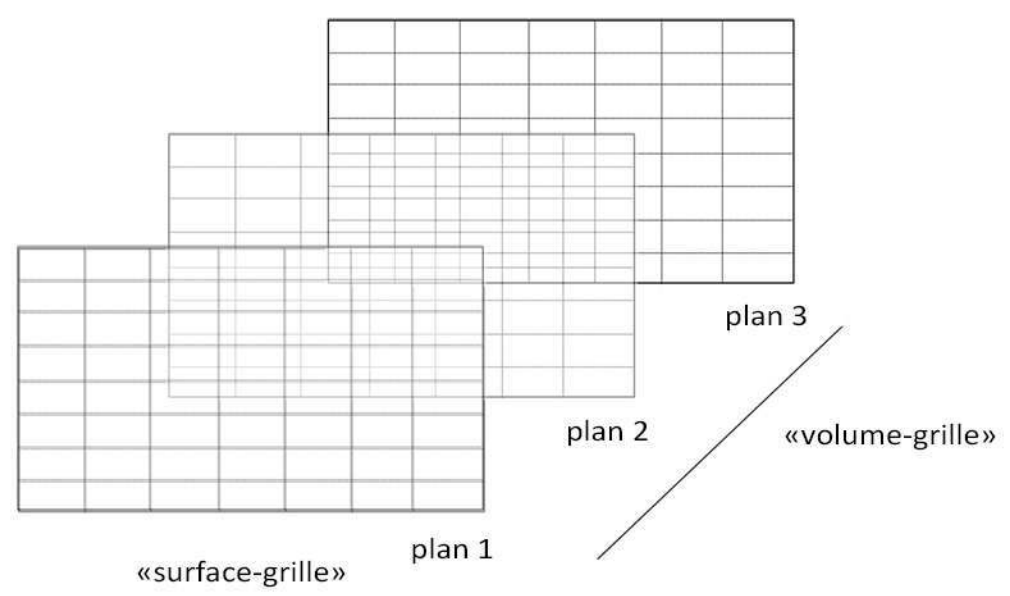

Figure 3 : Trois plans superposés accueillant chacun une grille

Avant d'appréhender plus précisément le rôle des «multi » dans la construction d'un webdocumentaire, nous devons évoquer l'application du multiplan et l'intégration du vecteur " profondeur » à l'élaboration d'une séquence et à la fragmentation du discours. Comment projeter le volume virtuel ${ }^{13}$ dans le processus de création, afin de l'actualiser dans les images résultantes et dans l'interactivité? Afin d'apporter un élément de réponse à cette interrogation, nous convoquerons une notion proposée par Edmond Couchot. Parmi plusieurs spécificités de la simulation 3D algorithmique, évoquées par cet auteur, nous en retiendrons une : «image puissance image ${ }^{14}$ ». Dans un premier temps, nous présenterons cette formule, puis dans un second temps, nous préciserons son application au domaine du webdocumentaire. Une première interprétation nous conduit dans le domaine des mathématiques (xn), mais dans notre cas, un chiffre est remplacé par un mot. Nous savons que l'élévation à une puissance est un facteur introduisant une multiplication. Ici, nous obtenons donc pour résultat une image multipliée par une image. Certes, nous remarquons que le préfixe multi est une nouvelle fois convoqué dans l'approche des objets numériques, mais il est nécessaire de présenter succinctement le 
processus de simulation pour apprécier la pertinence de la proposition. La simulation 3D implique une première étape, la modélisation, qui est à l'origine de l'engendrement d'une base de données contenant toutes les informations relatives à l'ensemble des parties constitutives de l'objet ou de la scène modélisés. Au cours d'une autre étape, afin d'obtenir une image 2D affichée sur une surface plane (l'écran), l'infographiste appliquera un point de vue à une caméra virtuelle. Quittons momentanément l'univers de la synthèse pour considérer la représentation photonique. En tant que continuum spatio-temporel, le réel ne peut être saisi filmiquement qu'au présent. Il est donc impossible de filmer ce qui n'a pas été capté lors du déroulement d'un événement. C'est précisément cette contrainte que la synthèse algorithmique nous propose de supprimer. Il est alors possible de projeter de multiples fois, au présent, un regard sur la base de données et ainsi, générer par calcul une nouvelle image correspondant aux choix de la représentation.

Maintenant, évoquons la convocation de cette notion pour appréhender le webdocumentaire, la construction d'un fragment de discours et l'interactivité. La simulation 3D propose une infinité de points de vue et la scène modélisée contient donc une infinité d'images en puissance. Nous allons rapprocher cette particularité du couple multiplan-profondeur que nous proposons. Chaque plan peut accueillir plusieurs éléments sources modifiés et l'image finale est donc le résultat d'un assemblage de fragments. La particularité de ce type de structure implique que la construction d'une infinité de combinaisons entre les fragments soit porteuse de variations relatives aux composantes de l'écriture filmique interactive. A la multiplication des possibilités de constructions visuelles et discursives, répond la multiplication des éléments de parcours inscrits dans la structure hypertextuelle. où sont affichés des fragments porteurs d'espace-temps différents. La présence simultanée de plusieurs temporalités, mais aussi de la représentation de lieux proches ou éloignés démultiplie les ressources liées aux développements du récit et de l'interactivité. Le fragment est un vecteur par l'intermédiaire duquel il est envisageable de penser de multiples combinaisons porteuses d'accélération, de pause ou encore de ralentissement, en ce qui concerne le temps et la vitesse. A propos de l'espace, nous évoquerons les possibilités de rapprochement et d'éloignement. Ces différentes tendances résultent de la juxtaposition de deux fragments, mais il est possible d'accroitre le nombre de fragments pour moduler la résultante.

La juxtaposition des fenêtres implique la coexistence de fragments d'origine et de type différents qui peuvent cohabiter temporellement suivant des modes propres aux écritures créées. Soit, par exemple, un plan filmique, nous pouvons l'afficher à l'arrière- 
plan de la construction et ainsi révéler l'ensemble des éléments saisis lors de la prise de vues. Il est aussi envisageable de pratiquer un recadrage, d'afficher le résultat dans une fenêtre et de cette façon, exclure une partie qui devient hors-cadre. Dans un second temps ou bien simultanément, il est possible d'afficher le précédent hors-cadre dans une deuxième fenêtre. Imaginons que nous intégrions un autre plan filmique en relation avec le premier et que nous pratiquions une approche similaire de découpage. Nous sommes alors en présence de quatre fenêtres, dont la gestion temporelle de l'affichage démultiplie les possibilités de liaison et de sens. Par ailleurs, nous disposons aussi de la possibilité de placer ces fenêtres sur des plans différents. Cette option aura pour effet d'autoriser un recouvrement intégral ou partiel d'une fenêtre, donc d'un fragment d'image par un autre. Ensuite, un déplacement entraînera une visibilité d'une fenêtre qui pourra dans son évolution se rapprocher et rencontrer une autre fenêtre, afin d'induire une modification $\mathrm{du}$ sens initial. Nous remarquerons aussi, à cette occasion, que des grilles différentes entrainent l'intégration de fenêtres de dimensions variables ${ }^{15}$. Nous noterons en outre que ces ressources sont aussi disponibles pour supporter les temps et lieux de l'interactivité. Cette présentation illustre les potentialités d'une telle structure, mais bien entendu, le dispositif est présent pour accueillir les éléments visuels et sonores et il s'estompe au profit d'une écriture. La complexité de la structure dépendra des types de narration retenus pour développer un thème.

Par l'intermédiaire du multifenêtrage, plusieurs points de vue, mais aussi plusieurs cadrages complémentaires d'une même scène peuvent être présents simultanément. Les fenêtres et leurs fragments visuels sont comme une composante paradigmatique dont de multiples alternatives seraient convoquées au même moment pour compléter, décupler des vecteurs de sens ou bien encore exister par leurs différences. Dans ce cas, les contraintes du découpage, du cadrage, du point de vue, du montage linéaire, des raccords entre les plans filmiques et du mono-fenêtrage sont partiellement déplacées. Dans l'univers du multifenêtrage, l'existence du raccord peut aussi apparaître à l'extérieur des fenêtres, dans les intervalles qui les séparent. Les «multi » introduisent de nouveaux régimes de vision et transforment la perception des événements.

Les fragments distribués sur les plans sont autant de lieux de la construction visuelle où progressent le récit, mais aussi la représentation des espaces convoqués pour exposer le thème du document, sans oublier l'interactivité, vecteur clé de l'écriture hypermédia. La présence des fragments liés aux composantes de cette construction est aussi porteuse d'écarts. Plus précisément, la création d'un fragment et sa présence impliquent des choix, donc des exclusions et des priorités qui sont inducteurs d'écarts. Nous retenons la notion d'écart pour appréhender ce qui est introduit et se forme entre deux ou plusieurs éléments. Dans ce cas, nous nous intéressons plus particulièrement à ce qui existe entre les fenêtres, et est toutefois dépendant mais aussi inducteur d'effets. Le multiplan et l'épaisseur des images, le multifenêtrage en tant que vecteur de modulation de la surface de l'image, la fragmentation et la multiplication des points de vue et la mobilité des fenêtres sont autant de vecteurs par lesquels s'opère le jeu des découpages, des remplissages et des écarts liés à l'élaboration de l'image résultante.

Le choix d'une option narrative ne conduit plus systématiquement à un renouvellement intégral de l'image. Un ensemble de nuances devient alors plus explicite et autorise la création de combinaisons plus souples et des articulations entre la navigation, la représentation et le récit. 


\section{Conclusion}

Nous nous sommes intéressés plus particulièrement aux spécificités de la forme numérique pour appréhender le webdocumentaire. Nous avons remarqué que l'intégration du numérique dans les processus de construction des images peut induire des structures relativement complexes, orientées vers l'élaboration interactive de la narration. Notre approche nous a conduit à constater que cette intégration est certes significative de l'hybridation avec le web, mais que de nombreuses pistes demeurent à découvrir pour étendre et renouveler les écritures et proposer aux internautes de nouvelles options de découverte d'un objet interactif.

Par ailleurs, nous noterons que les couples surface-grille et volume-grille ne constituent que la base de la construction de structures induisant la génération d'images composites, où chaque fragment joue un rôle. Cette organisation visuelle est au service de la déclinaison de la narration, de la navigation et de l'interface entre l'espace-temps de l'internaute et celui de la représentation du réel propre aux thèmes développés.

Il demeure également indispensable de procéder à des détours pour accorder un temps de réflexion aux développements du genre documentaire, mais aussi au cadre d'investigation proposé par l'anthropologie visuelle, sans oublier la capacité d'ouverture des auteurs de bandes dessinées à interroger leur art.

\section{BIBLIOGRAPHY}

Prison valley $:$ http://prisonvalley.arte.tv/?lang=fr

Pékin sans transition : http://pekin.franceculture.fr/

Irak - 10 ans - 100 regards : http://irak.arte.tv/mosaique/

Jeu d'influences : http://jeu-d-influences.france5.fr/

La lèpre toujours d'actualité : http://www.raoul-follereau.org/webdoc_jml_2015/\#Accueil

IMAROC art contemporain : http://www.imaroc-contemporain.org/

The defector - escape from north korea : http://thedefectormovie.com/index.php

Pekin underground : http://www.pekinunderground.com/\#/home

Diplomates : http://webdocs.diplomatie.gouv.fr/diplomates/\#poster

Voyage au bout du charbon : http://www.lemonde.fr/asie-pacifique/visuel/2008/11/17/voyage-aubout-du-charbon_1118477_3216.html

Thanatorama : http://www.thanatorama.com/

La zone, Retour à Tchernobyl : http://www.lemonde.fr/week-end/visuel/2011/04/22/la-zone-

retour-a-tchernobyl_1505079_1477893.html

World brain : http://worldbrain.arte.tv/\#/

Planet solar : http://future.arte.tv/fr/planet-solar

Polar Sea $360^{\circ}:$ http://polarsea360.arte.tv/fr/

Fort Mc Money : http://fortmcmoney.com/\#/fortmcmoney

Alma - une enfant de la violence : http://alma.arte.tv/fr/

Le Challenge : http://www.canalplus.fr/c-infos-documentaires/pid3400-c-le-challenge.html?nav=1 
Les voyageurs : http://lesvoyageurs.medecinsdumonde.org/?

gclid=CMS49MCo-8QCFaTnwgodXYcALA\#Prehome

Transkraïn : http://www.lemonde.fr/europe/visuel/2014/02/07/transkraina-aux-confins-de-l-

ex-urss_4358998_3214.html

Grriottes Girrls : http://rdchezlesgauloises.free.fr/\#_Intro

Veduta : http://www.therabbithole.fr/2013/11/29/veduta-un-webdocumentaire-sur-lart-

contemporain/

Duchoramas : http://www.therabbithole.fr/2013/11/29/veduta-un-webdocumentaire-sur-lart-

contemporain/

Les filles de Gerland : http://www.rue89lyon.fr/les-filles-de-gerland/

Iran au régime des sanctions $:$ http://webdoc.france24.com/iran-sanctions/

Bibliographie

Françoise Holtz-Bonneau, Création infographique, Paris, Addison-Wesley, 1994.

Edmond Couchot, «Image puissance image », Revue d'esthétique, Images, nouvelle série $n^{\circ} 7,1984$, pp. 123-133.

Nicolas Bole et Cédric Mal, Le webdoc existe-t-il ?, Le blog documentaire, 2014.

Lev Manovich, Le langage des nouveaux médias, Dijon, Les presses du réel, 2010.

François Niney, Le documentaire et ses faux-semblants, Paris, Klincksieck, 2009.

René Prédal, Esthétique de la mise en scène, Paris, Cerf-Corlet, 2007.

\section{NOTES}

1. Expression dédiée qui traduit l'effet de modernité attendu dans la production d'images lors d'un avènement technique. Cependant, l'observation de la production révèle que l'intégration de nouveaux outils et processus n'est pas une condition suffisante pour générer des évolutions pertinentes. Une perspective réflexive s'impose pour transférer et renouveler des savoir-faire qui s'articulent avec une approche créative, où la mise en œuvre des spécificités des nouveaux dispositifs est au centre des projets.

2. - Le mode composition: «De part sa constitution technique naturelle, l'image informatique est une image composée ou recomposée: tous ses éléments sont répertoriables, définissables et accessibles séparément, du moins théoriquement. (...) Puisque l'image infographique est, si l'on se situe du côté de la conception et de la réalisation, bâtie sur un mode composé, c'est bien à partir de cette nature là que peuvent se situer les enjeux de la création. » (Françoise Holtz-Bonneau, Création infographique, Paris, Addison-Wesley, 1994, p. 70).

- Le mode mutation : La modification est le principe même de la mutation. Elle relève des " possibilités transformationnelles de l'infographie » appliquées à une image initiale. « La mutation est encore plus fondamentalement un mode lié au mouvement. (... ) Les divers modes de mutation [sont] les mutations concernant les attributs des formes [luminance, chrominance et texture] et les mutations concernant les formes elles-mêmes [anamorphose et métamorphose].» (ibid., p. 100-104).

- Le mode déambulation : « Déambuler n'est pas seulement flâner ou musarder, c'est aussi rester ouvert à une grande souplesse de parcours et de pause. [ ] C'est rester disponible pour toute surprise et toute aventure. » (ibid., p. 118). 
3. Dans le cas de l'image « entière » la surface de l'image source est égale à celle de l'écran qui l'accueille. Par exemple, dans un processus de traitement, l'image vidéographique est considérée comme « entière » si elle occupe l'intégralité de l'écran d'affichage.

4. L'image résultante présente une surface qui pourra être inférieure à celle de l'écran et elle est obtenue par l'application d'un processus où sont réunies une ou plusieurs sources visuelles. Dans un premier temps, nous privilégions la dimension visuelle pour des raisons méthodologiques, mais la composante sonore n'est pas exclue car elle est partie intégrante du projet créatif du webdocumentaire.

5. La création intègre tant la modification d'image photonique que la genèse d'image algorithmique.

6. Le terme "plan » est employé pour désigner un lieu spatial lié à la construction de l'image, alors que le résultat d'une saisie filmique sera désigné par l'expression «plan filmique».

7. Nous évoquons l'image car actuellement nous considérons la dimension spatiale de l'objet construit. Mais l'image sera ensuite appréhendée en tant qu'élément du plan filmique par l'intermédiaire duquel la composante temporelle se développe.

8. Ce terme exprime la capacité d'un dispositif technique de montage numérique ou de création par compositing, à permettre un découpage temporel des opérations de modification et d'assemblage de fragments visuels sur les différents plans de l'image en cours de création.

9. Auteurs : David Dufresne et Philippe Brault / Thème : enquête journalistique relative à l'économie carcérale américaine de la ville de Canon City - Comté de Frémont - Colorado / 2010.

10. Les auteurs ne juxtaposent pas simultanément plus de trois fragments visuels (sauf dans un cas où six portraits s'affichent progressivement), certainement pour éviter une saturation du sens.

11. Nous ne retenons pas le terme « tridimensionnel » car il s'inscrit prioritairement dans la définition spatiale du réel, qui n'est qu'un aspect de la composante " tridimension ». En l'occurrence, la troisième dimension n'est pas exclusivement spatiale puisqu'orientée vers l'investigation de l'interactivité et vers la notion d'épaisseur, liée à la superposition des plans et des fragments qu'ils accueillent.

12. La grille est un outil activé dans la mise en page des supports papier et électronique.

13. Nous rappelons que la combinaison des «multi » et du vecteur « profondeur » induit l'existence virtuelle d'un volume.

14. Edmond Couchot, «Image puissance image », Revue d'esthétique, Images, nouvelle série $\mathrm{n}^{\circ} 7,1984$, p. 123.

15. L'évocation de ces combinaisons liées aux «multi » est le résultat, d'une part, d'une réflexion et, d'autre part, de l'intégration des productions d'artistes vidéo qui ont commencé, à partir des années 1985, à expérimenter la vidéo numérique avec des magnétoscopes et des palettes graphiques. Certes, l'interactivité n'appartenait pas à ces réalisations mais une expérimentation plastique était à l'œuvre. 


\section{ABSTRACTS}

The webdocumentary has, among its characteristics, the combination of the specificities of the two components that make up its name. The integration of the digital form by the documentary and its close links to the internet media have given birth to new forms of written storytelling which can either revisit the documentary genre or open up onto the powerful opportunities offered by data exchange and social networking. The present paper will examine the trends fostered by the production of webdocumentaries; the current investigation will take into account the history of the study of visual forms introduced by digital videography and the inception of the hypermedia and of algorithmic images.

In the past, the renewal of the automatic and technical devices associated with cinema, analog videography and with computer graphics has led to the reproduction of the dominant trends and to the marginal development of new forms of writing. The present study will try and see whether the hybridization of both the authenticated genre of the documentary and of the Web has favoured closer links between mainstream and marginal forms and whether it has questioned the original genres and prioritised the emergence of new creative "principles".

Le webdocumentaire a pour caractéristique d'intégrer les particularités de ses deux composantes. L'intégration de la forme numérique par le documentaire et son rapprochement du média internet induisent donc de nouvelles ressources d'écriture. Celles-ci peuvent revisiter le genre documentaire, mais aussi s'ouvrir à la puissance des réseaux informatiques et sociaux. Nous proposons de repérer les tendances de la production de webdocumentaires en inscrivant notre investigation dans la continuité de l'histoire des régimes de vision introduits par la vidéographie numérique, et l'avènement de l'hypermédia et des images algorithmiques.

Dans le passé, un renouvellement des dispositifs techniques automatiques liés au cinéma, à la vidéographie analogique et à l'infographie, a entraîné une reproduction des tendances dominantes et un développement marginal de nouvelles écritures. Nous chercherons à repérer si l'hybridation d'un genre confirmé, le documentaire, et du web, a favorisé le rapprochement de la marge vers le centre, en remettant en cause les écritures initiales et en favorisant l'émergence de nouveaux « principes » créatifs.

\section{INDEX}

Mots-clés: Mots-clés : multifenêtrage, multiplan, multicouche, tridimension, contrôle de la surface, image " résultante ». 